\title{
Lusioersily
}

\section{Interval type-2 fuzzy logic based multiclass ANFIS algorithm for real-time EEG based movement control of a robot arm}

Bhattacharyya, S., Basu, D., Konar, A., \& Tibarewala, D. N. (2015). Interval type-2 fuzzy logic based multiclass ANFIS algorithm for real-time EEG based movement control of a robot arm. Robotics and Autonomous Systems, 68, 104-115. https://doi.org/10.1016/j.robot.2015.01.007

Link to publication record in Ulster University Research Portal

Published in:

Robotics and Autonomous Systems

Publication Status:

Published (in print/issue): 30/06/2015

DOI:

10.1016/j.robot.2015.01.007

\section{Document Version}

Author Accepted version

\section{General rights}

Copyright for the publications made accessible via Ulster University's Research Portal is retained by the author(s) and / or other copyright owners and it is a condition of accessing these publications that users recognise and abide by the legal requirements associated with these rights.

\section{Take down policy}

The Research Portal is Ulster University's institutional repository that provides access to Ulster's research outputs. Every effort has been made to ensure that content in the Research Portal does not infringe any person's rights, or applicable UK laws. If you discover content in the Research Portal that you believe breaches copyright or violates any law, please contact pure-support@ulster.ac.uk. 


\title{
Interval Type-2 Fuzzy Logic based Multiclass ANFIS Algorithm for Real- Time EEG based Movement Control of a Robot Arm
}

\author{
Saugat Bhattacharyya ${ }^{1,2}$, Debabrota Basu ${ }^{1}$, Amit Konar ${ }^{1}$, D.N. Tibarewala ${ }^{2}$ \\ ${ }^{1}$ Dept. of Electronics \& Telecommunication Engineering, ${ }^{2}$ School of Bioscience \& \\ Engineering, Jadavpur University, Kolkata, India-700032 \\ E-mail-saugatbhattacharyya@live.com, basudebabrota29@gmail.com, \\ konaramit@yahoo.co.in, biomed.ju@gmail.com
}

Corresponding author: Saugat Bhattacharyya

Postal Address: School of Bioscience and Engineering,

Jadavpur University,

Raja S.C. Mullick Road,

Jadavpur, Kolkata

India-700032

Contact No.: +91-9932866197

E-mail: saugatbhattacharyya@live.com

\begin{abstract}
:
Brain-computer interfacing is an emerging field of research where signals extracted from the human brain are used for decision making and generation of control signals. Selection of the right classifier to detect the mental states from electroencephalography (EEG) signal is an open area of research because of the signal's non-stationary and Ergodic nature. Though neural network based classifiers, like Adaptive Neural Fuzzy Inference System (ANFIS), act efficiently, to deal with the uncertainties involved in EEG signals, we have introduced interval type-2 fuzzy system in the fray to improve its uncertainty handling. Also, real-time scenarios require a classifier to detect more than two mental states. Thus, a multi-class discriminating algorithm based on the fusion of interval type-2 fuzzy logic and ANFIS, is introduced in this paper. Two variants of this algorithm have been developed on the basis of One-Vs-All and One-Vs-One methods. Both the variants have been tested on an experiment involving the real-time control of robot arm, where both the variants of the proposed classifier, produces an average success rate of reaching a target to $65 \%$ and $70 \%$ respectively.
\end{abstract}


The result shows the competitiveness of our algorithm over other standard ones in the domain of non-stationary and uncertain signal data classification.

Keywords - Real-time control, Interval type-2 fuzzy system, Adaptive neural fuzzy inference system, Multi-class classification, Motor imagery

\section{INTRODUCTION}

Human-machine interaction (HMI) [1] is rapidly evolving as a potential field of research in applied biomedical and cognitive science. In this paper, we have dealt with an emerging trend of HMI called brain-computer interfacing (BCI), where the user interacts with a computing device or robot directly through mental intentions (or commands), generated as signals, from the brain [2].

A BCI technology is broadly composed of four basic processes, viz., recording the mental activity (Signal Acquisition); extraction of the intended action or desired features from that activity (Signal Processing); generation of the desired action (Mental state detection); and feedback, either through intact sensation, such as vision, or generated and applied by the prosthetic device (Feedback) [3]. Each of the aforementioned processes requires highly efficient techniques of signal processing, machine learning and control theory whose functions are to unveil the information embedded within the brain signals for various applications, like in robotics, communication, and gaming [4-7]. But BCI will be most helpful in neuro-rehabilitation [8,9] of physically challenged patients, like those suffering from paralysis, Amyotropic Lateral Sclerosis, cerebral palsy, loss of limb [10]. These brain signals are extracted, decoded and studied with the help of various brain measures like Magnetoencephaography, functional Magnetic Resonance Imaging, Electro-corticography, and Electroencephalography (EEG) $[11,12]$. In our analysis, we have preferred to use EEG signal over other measures because it is portable, easy to use, inexpensive, and has a higher temporal resolution $[10,13]$.

For every cognitive task performed by the user, a characteristic brain modality is generated from the brain at different locations. A BCI technology aims at decoding these brain modalities to control a robotic device and the selection of brain modalities for a specific control task is an important issue in BCI research. Examples of few frequently used modalities are steady-state visually evoked potential (SSVEP), slow cortical potential (SCP), P300, event related desynchronization/synchronization (ERD/ERS) and error related potential (ErRP) [10, 15]. In the current study, we aim to control the movement of a robot 
arm using five motor imagery mental commands: Forward, Backward, Left, Right and No movement. Using these commands the subject would attempt to move the arm towards a randomly positioned target (placed within the reach of the robot arm). ERD/ERS signals originates during movement planning, movement imagination or movement execution (collectively, referred to as motor imagery signals) $[15,16]$. Thus, this modality have relevance for control purpose in our present study.

In this paper, we have also delineated the importance of multiclass classification $[17,18]$ in real world problems and how it can be employed efficiently. In real time scenarios, we often come across situations, which requires the classifier to detect more than one mental states. So in case of BCI systems, multiclass classification is quite important and has a wide scope of usage.

The brain signals recorded using EEG are non-linear, complex, non-stationary and nonGaussian. Thus, they are quite challenging to classify and the problem is nothing but a conundrum. Adaptive neural fuzzy inference system (ANFIS) is a neural network [19, 20] inspired classifier, which is used to classify complex datasets using fuzzy inference systems. ANFIS, is a strong and standard neural fuzzy inference tool but due to its Type-1 fuzzy membership pattern, it fails to handle noise and uncertainty in case of chaotic and Ergodic signals. Also, ANFIS is dependent and sensitive to the parameter sets defined by the user [19]. These shortcomings of the classical ANFIS algorithm inspired us to associate type-2 fuzzy [21, 22] sets with classical ANFIS for BCI application.

In this paper, we have proposed two novel classification method based on the fusion of interval type-2 fuzzy system with the ANFIS structure for multiclass classification. In classical multiclass literature, 'one vs all' and 'one vs one' methods [10] are commonly used among researchers and these methods amalgamates the results of smaller binary classifiers to give the final hyperplane. Here, we have used ANFIS architecture for each of the binary classifiers and then the outputs of each individual binary classifiers are combined using a type-2 fuzzy to yield the final output.

Here, the EEG features are classified using our proposed type-2 fuzzy sets with the fuzzy inference system of ANFIS to minimize the adverse effects of uncertainty. This has made our algorithm a better tool to handle and classify EEG signals. It is more robust, efficient, user independent and handles the uncertainty of EEG signals much better than the previous model (classical ANFIS). Our proposed classifier also shows its competitiveness to discriminate between multiple classes as compared to other state-of-art classification algorithms. 
The rest of the paper is arranged as follows. In section 2, we describe the acquisition system and the robot arm used in this paper. In section 3, we discuss on the experimental and data processing techniques used for offline classification and online control of the robot arm. Our proposed multiclass ANFIS networks and their working procedures are described in section 4 of this paper. A discussion on the results of the offline and online experiments using our proposed classification algorithm are mentioned in section 5 , followed by the concluding remarks in section 6 .

\section{MATERIALS AND CONTROL METHODS}

In this study, the subject controls the movement of a Jaco robot arm [23, 24] using five motor imagery signals related to following movement states: forward, backward, left, right and no movement. This section gives a brief background on the EEG acquisition system and the Jaco Robot arm, followed by a discussion on the control strategy implemented in this study.

\subsection{EEG Data Acquisition System: Emotiv Epoc}

The mental states of the users in form of EEG signals are recorded using an Emotiv Epoc System. It is a high resolution, multi-channel, wireless neuro-headset which uses a set of 14 sensors (electrodes) and 2 references. The electrodes are arranged according to the standard 10-20 electrode system [25] and their locations are AF3, F7, F3, FC5, T7, P7, O1, O2, P8, T8, FC6, F4, F8 and AF4 (Fig. 1). The sampling rate of the EEG system is $128 \mathrm{~Hz}$ with a resolution of $0.51 \mu \mathrm{V}$. The system comprises of a built-in digital $5^{\text {th }}$ order sinc filter with a bandwidth of $0.2-45 \mathrm{~Hz}$ and a digital notch filter at 50 and $60 \mathrm{~Hz}$.

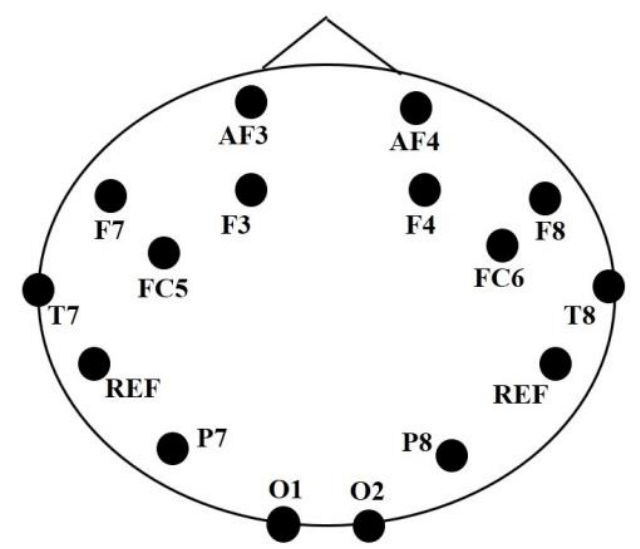

Fig. 1. Electrode locations in the Emotiv Epoc system 


\subsection{Jaco Robot Arm}

Jaco Robot Arm, developed by Kinova, is a 6-axis robotic manipulator with a three fingered hand. The arm has six degrees of freedom in total with a maximum reach of $90 \mathrm{~cm}$ radius sphere and maximum speed of $30 \mathrm{~cm} / \mathrm{sec}$. It is made of three sensors: force, position and acceleration. This arm is suitable for a person with a disability of the upper arm and can be placed on a wheelchair. The upper arm of the robot is made of three links which is similar to the upper limb of the human body, as shown in Fig. 2. An API is provided from the manufacturers which allows greater freedom of control by users [23, 24].

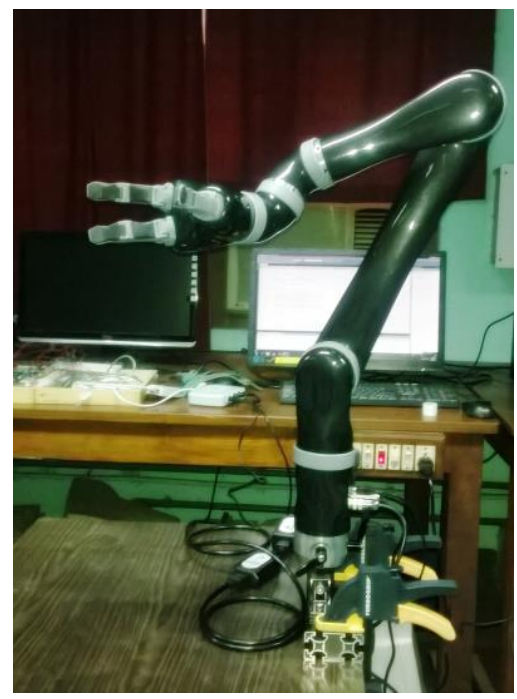

Fig. 2. Jaco Robot Arm setup in our lab

\subsection{Online Control Scheme}

As mentioned earlier, the subject needs to control the movement of the robot arm towards a given target by using five mental (motor imagery) commands: Forward (F), Backward (B), Left (L), Right (R) and No movement $(\mathrm{N})$. To stop the movement of robot arm, the subject would generate a No Movement command by relaxing. The rest of the commands are employed to move the robot arm in their respective directions. For example, if the subject wants to move the robot arm in the forward direction, he would need to imagine moving forward, which would generate a forward command from the brain signals. The control signals generated according to the mental commands are given in Table 1.

The control scheme, as shown in Fig. 3, requires the subject to first observe the current position of the end-link of the robot arm with relation to the target position and then would plan the next movement of the robot arm. The motor imagery signals thus generated are acquired by the Emotiv acquisition system. Next, the acquired EEG signals are pre-processed to remove any noise present in the data. Then a feature extraction algorithm is applied to the filtered data to construct the feature vector. The feature vector consists of specific 
information about the different mental commands, which are based on their characteristic ERD/ERS waveform. This feature vector is then fed to the classifier to decode the mental state of the subject. The decoded output is used to generate the control signal (as shown in Table 1) to move the robot arm in the required direction.

Table 1. Control signals generated to move the robot arm according to mental command generated by the subject

\begin{tabular}{ll}
\hline Mental Command & Control signal \\
\hline Forward & Move robot forward by 10 units \\
Backward & Move robot backward by 10 units \\
Left & Turn robot in counter-clockwise direction by $10^{\circ}$ \\
Right & Turn robot in clockwise direction by $10^{\circ}$ \\
No Movement (Relax) & Stop robot movement
\end{tabular}

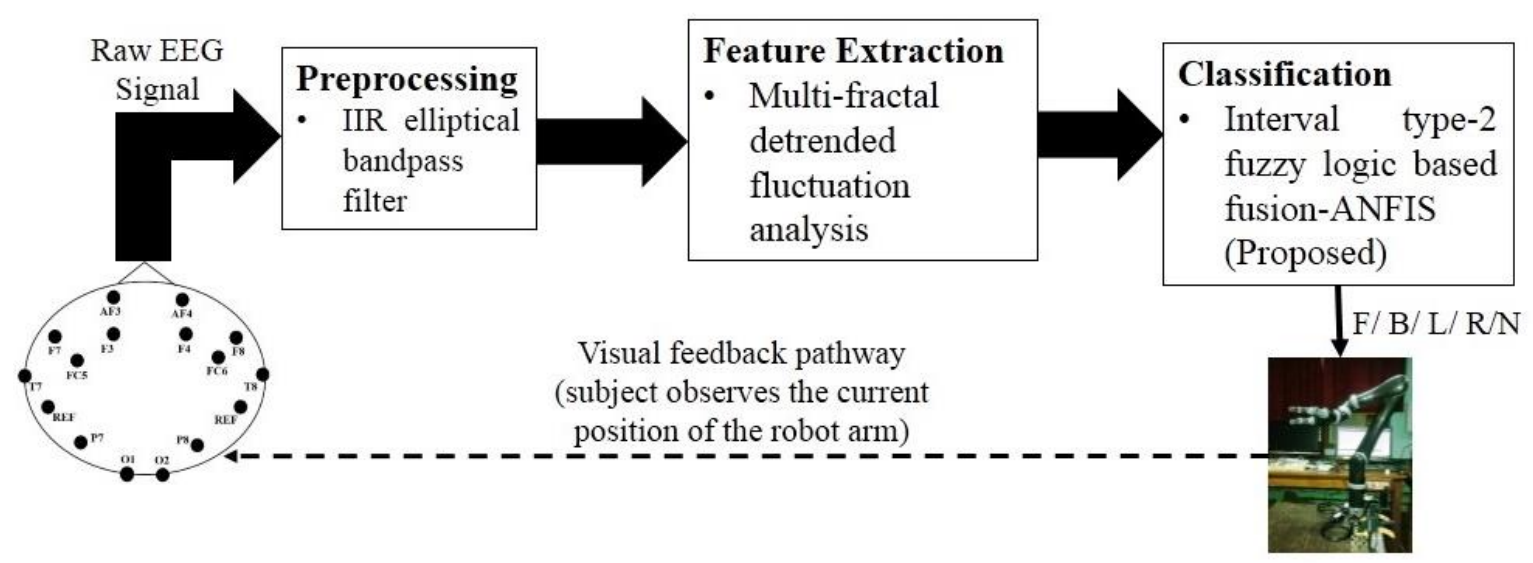

Fig. 3. Block diagram of the online control scheme (Abbreviation: F- Forward, B-Backward, L-Left, R-Right, N-No movement).

\section{EXPERIMENTS AND DATA PROCESSING}

The first step towards movement control of the Jaco robot arm is for the subject to undergo training. During this phase, the subject trains itself to generate mental commands needed to control the robot arm movement. Also, the dataset accumulated during the training period is used to train the classifier. Based on this training, the classifier produces one of the five mental commands as outputs which is further used to generate the control signals.

Eleven right-handed subjects (6 female and 5 male) with normal vision and no prior disability or illness have participated in this study. The experiment would require the EEG signals to be free from any other unknown environmental stimulus (noise), so the subjects would imagine kinesthetic tasks based on a visual stimuli projected on a screen in a well-lit, empty, sound-proof, isolated room. 
Prior to data acquisition, the subjects is informed of the various details of the experiment. Then, the subjects are required to fill and sign a consent form. The procedures of the experiments conducted abide by the requirements set in the Helsinki Declaration of 1975, revised in 2000.

This section describes the experimental and data analysis techniques applied to undertake the training procedure along with the details on online experimentation implemented in this study.

\subsection{Stimuli Generation}

A visual stimuli is used in this study to provide instructions to the subject on the mental task he has to perform during the training phase. The visual cue contains instructions for five mental commands: Forward, Backward, Left, Right and No movement, in form of direction of an arrow, as shown in Fig. 4.

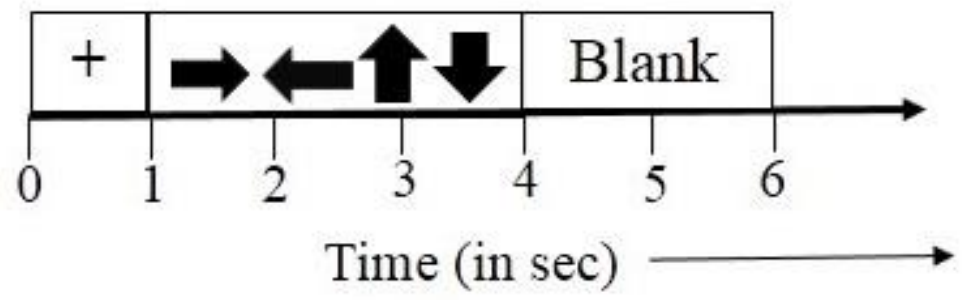

Fig. 4. Timing diagram of a motor imagery trial performed by the subject. The direction of the arrows provides instruction to the subject.

The training of each subject is undertaken over seven different sessions and one session is performed on a single day. It had been observed that by 7 sessions, each subject had produced a consistent EEG response, which resulted in obtaining a training accuracy of more than $80 \%$. Each session comprises a total of 100 repetitive trials where each mental tasks is repeated 20 times. The generic timing structure of each trial is shown in Fig. 4. First, a fixation ' + ' is displayed on screen for one second which is an instruction for the subject to get ready. Next, the task instructions in form of arrows are displayed on screen randomly for 3 seconds. During this period the subject mentally performs the task given. For generation of the 'No movement' command, a blank screen is displayed in place of the arrows. Then, a blank screen is displayed for 2 seconds during which the subject is allowed to relax. This period also prevents the overlapping of consecutive mental states on the EEG signal.

\subsection{Data Preprocessing}

ERD/ERS modalities, generated during mental imagery tasks, are found to be prevalent in the $\mu(8-12 \mathrm{~Hz})$ and central $\beta(16-24 \mathrm{~Hz})$ bands $[10,25]$. For this purpose, we have designed an elliptical band-pass filter of order 12 , pass-band attenuation of $1 \mathrm{~dB}$ and stop-band attenuation 
of $50 \mathrm{~dB}$ to extract movement related information in the bandwidth of $8-25 \mathrm{~Hz}$ from the raw EEG signals. Also, this step allows the removal of environmental and cognitive noises (background EEG) from the signal. An elliptical filter is used because of its equi-ripple behavior in the pass-band and stop-band and has a steeper roll-off characteristic when compared to other standard filters [26].

After the filtering step, during offline training phase, the EEG data pertaining to 3 seconds of motor imagery tasks are extracted from each trial for further processing. Each 3 seconds of data are further partitioned into 500 milliseconds data vectors, on which further processing is performed. But during online experimentation, after incoming EEG is filtered, data vectors of 500 milliseconds before the current time is created for processing. Then the data in both offline and online cases is normalized to the interval $[0,1]$.

\subsection{Feature extraction using multi-fractal detrended fluctuation analysis}

If a time-series signal repeats itself on the subintervals of the signal, then it possesses scale invariant structures. For EEG signals, the scale invariant structures of inter-spike interval of firing of the neurons are capable of discriminating between the neural activities of brain. Alterations in scale invariant structure of bio-signals indicate adaptability of physiological processes which can be quantified using Detrended fluctuation analysis (DFA) [27-28]. Another salient feature of DFA is that it is not affected by non-stationarity of a signal and can measure long range correlations of such signals. But time series with complicated temporal behavior necessitate different scaling exponents for different part of the series. In such case multi-fractal analysis is performed which provides multiple scaling exponents to completely describe the behavior of the time series for different scaling parameters. Thus, mutli-fractal detrended fluctuation analysis (MFDFA) allows the formalism of non-stationary signals for characterization of the time series [27-28]. The steps required to calculate the MFDFA estimates are summarized below:

Let, $x_{k}$ is a time series of length $N$ of compact support that $x_{k}=0$ for an insignificant amount of values.

I. First, compute the sequence of summary displacements (Profile) $P(i)$ by

$$
P(i)=\sum_{k=1}^{i}\left[x_{k}-\bar{x}\right], i=1, \ldots, N
$$

II. Then, partition $P(i)$ in a number of non-overlapping segments denoted by $N_{l}=N / l$, of equal length $l$. The same process is repeated from end to start to 
the series to consider the small parts that can remain at the end of the series. Thus we obtain total $2 N_{l}$ segments.

III. In this step, detrend the profile $P(i), i=1, \ldots, N$, for each segment of length $l$, by applying least square fit on each segment and calculating their respective variance, which is given as

$$
\begin{aligned}
& F^{2}(l, v)=\frac{1}{l} \sum_{i=1}^{l}\left\{P[(v-1) l+i]-y_{v}(i)\right\}^{2} \\
& \text { where } v \text { is a segment such that } v=1, \ldots, N_{l}, \text { and } \\
& \mathrm{F}^{2}(l, v)=\frac{1}{l} \sum_{i=1}^{l}\left\{P\left[N-\left(v-N_{l}\right) l+i\right]-y_{v}(i)\right\}^{2}
\end{aligned}
$$

for $v=N_{l}+1, \ldots, 2 N_{l}$, where $y_{v}(i)$ is the fitting polynomial in the segment $v$.

IV. Then, calculate the $q^{\text {th }}$ order fluctuation function by averaging over all segments, as follows

$$
F_{q}(l)=\left\{\frac{1}{2 N_{l}} \sum_{v=1}^{2 N_{l}}\left[F^{2}(l, v)\right]\right\}^{q / 2}
$$

where $q$ can take any value other than zero. To determine the dependency of generalized $q$ dependent fluctuations on time scale $l$, repeat steps II to IV.

V. Lastly, determine the scaling behavior of the fluctuating functions by analyzing the $\log$-log plots of $F_{q}(l)$ versus $l$ for each value of $q$.

$$
F_{q}(l) \square l^{h(q)}
$$

Where, $h(q)$ is the q-dependent generalized Hurst exponent. [27]. It is to be noted that for long-range power-law corrected series $x_{i}, F_{q}(l)$ increases as powerlaw for large values of $l$. 

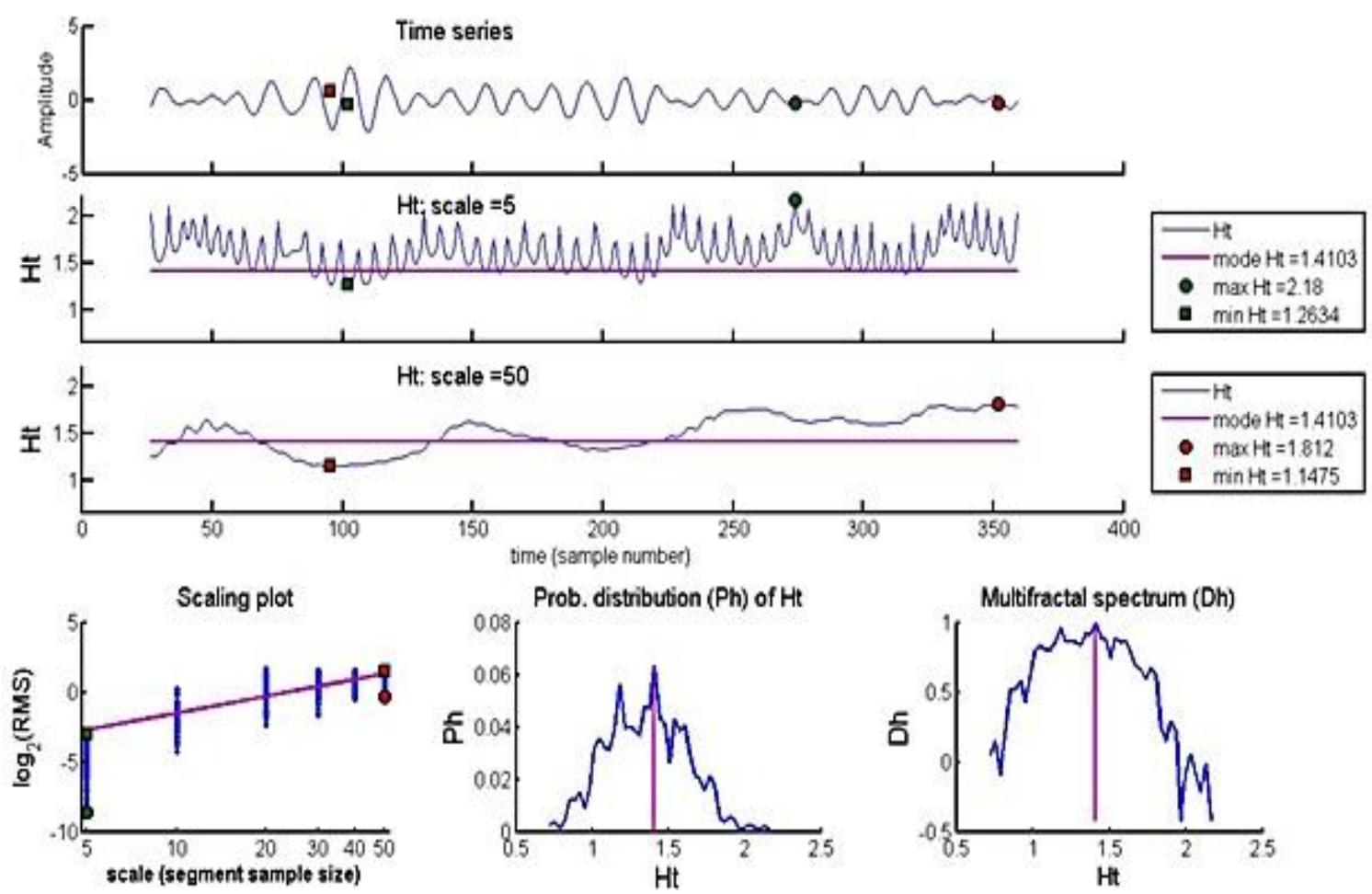

Fig. 5. An example of the Local Hurst Component (Ht), Probalility distribution ( $\mathrm{Ph}$ ) and Multifractal spectrum (Dh) of a motor imagery signal from electrode location FC5.

In this study, we have used MFDFA of $3^{\text {rd }}$ order fitting polynomial (i.e. $y_{v}(i)$ ) and varied $q$ in the range -5 to 5 with 101 discreet intervals. An example of the $3^{\text {rd }}$ order local Hurst components $(\mathrm{Ht})$, probability distribution of local Hurst components $(\mathrm{Ph})$ and multifractal spectrum of local Hurst components (Dh) from electrode location FC5 is illustrated in Fig. 5. The feature vector is prepared from the probability distribution of Hurst components and its dimension for both offline and online experimentation is 45 (for each electrode).

\section{TYPE-2 FUZZY BASED MULTI-CLASS ANFIS ALGORITHM}

This section begins with background descriptions of multi-class classification, ANFIS architecture and interval type-2 fuzzy system. The final sub-section describes our proposed algorithm which is a combination of the three methods.

\subsection{Multiclass Classification}

In real world problems, we often face situations, where the observations may belong to more than two classes unlike the binary classification or the dichotomies. Under this kind of scenario a training data point may belong to one of the $N$ different classes and one's aim is to find an approximation function $f$ of a classifier so that it can predict accurately for an unknown entry to which of the $N$ classes it belongs. 
There exist two widely used state-of-art approaches for the multiclass classification problems, which are quite apparent and trivial. The first one is one vs. all (OVA) classification approach and the other is one vs. one (OVO) classification approach.

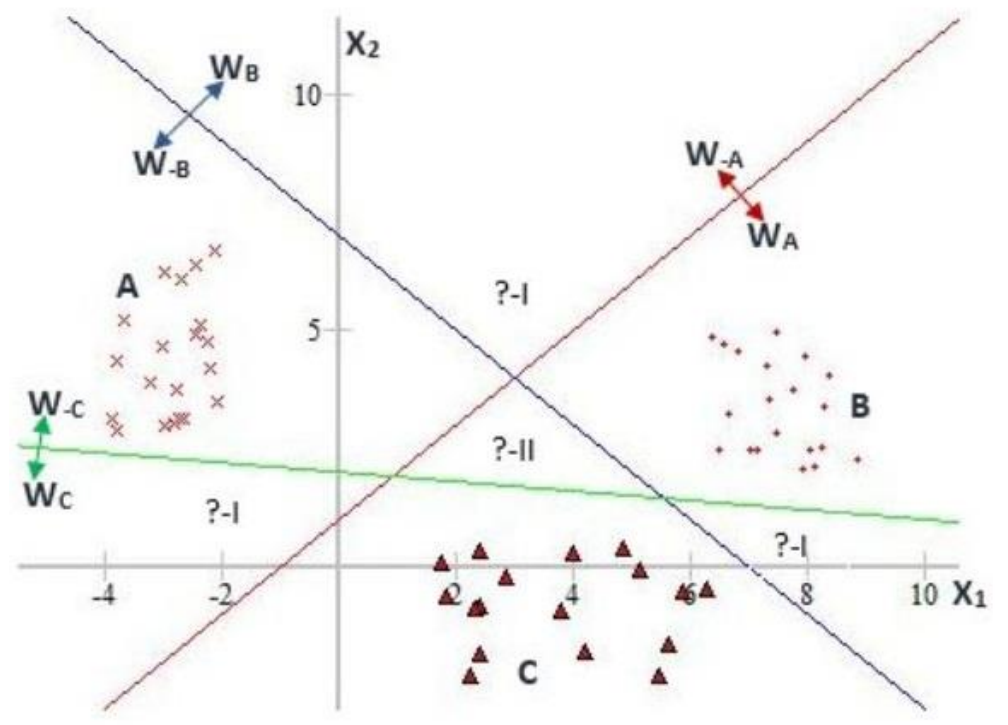

Fig. 6. Multi-class classification using OVA $(\mathrm{N}=3)$

In OVA approach (Fig. 6), we build $N$ different classifiers where each classifier corresponds to each individual classes. This approach reduces the problem into $N$ binary classification problems, where each of the binary classifiers discriminates a given class from rest of the classes [10,29]. For example, the $i^{\text {th }}$ classifier will give positive results for the data points belonging to the $i^{\text {th }}$ class and negative result for data points belonging to the other $N-1$ classes. If $f_{i}$ is hyperplane for the $i^{\text {th }}$ classifier, the final multiclass classifier hyperplane function can be defined as,

$$
f(\vec{x})=\arg \max _{i \in\{1, ., N\}} f_{i}(\vec{x})
$$




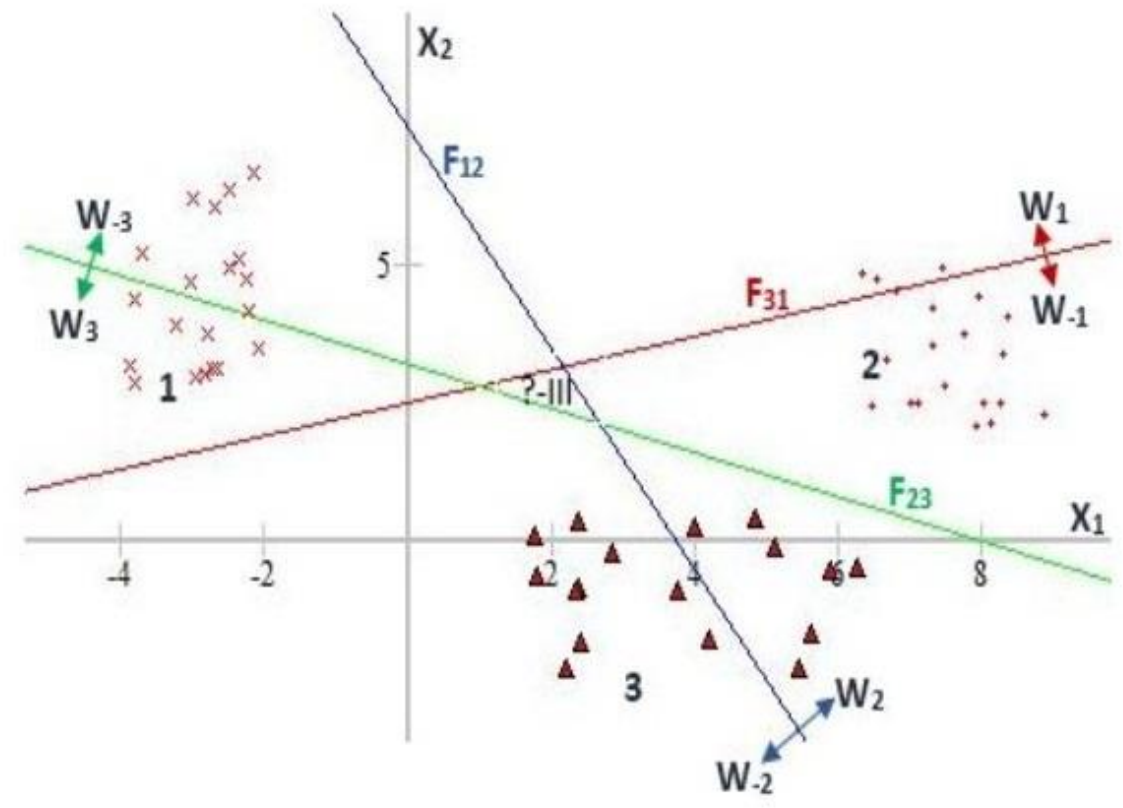

Fig. 7. Multi-class classification using OVO $(\mathrm{N}=3)$

In OVO approach (Fig. 7), $N(N-1) / 2$ binary classifiers to discriminate between data points of $i^{\text {th }}$ and $j^{\text {th }}$ classes $[10,29]$. Here, the classifier between $i^{\text {th }}$ and $j^{\text {th }}$ classes can be defined by the hyperplane $f_{i j}$, where $f_{i j}$ gives positive output for data points belonging to class $i$ and negative result for data points in class $j$. Thus the multiclass problem can be defined as finding a hyperplane function $f(\vec{x})$ such that,

$$
f(\vec{x})=\arg \max _{i}\left(\sum_{j} f_{i j}(\vec{x})\right)
$$

One of the basic problem faced by these methods is the way the piecewise hyper-planes constructed by individual OVO and OVA are fused to generate the final output hyper-plane. But inefficient fusions often cause discrepancies and high computational complexity. Thus, in this study, we have proposed a simple yet efficient approach of incorporating outputs of individual dichotomies of OVA and OVO using type-2 inference system, which will be explained in the final sub-section.

\subsection{Adaptive Neural Fuzzy Inference System (ANFIS)}

ANFIS is a very popular and efficient adaptive neural network and fuzzy based algorithm used in classification problems. It was first proposed by Jang in [19] based on the adaptive neural network structures and Takagi-Sugeno model based fuzzy inference systems [30].

In the ANFIS model, the neuro-fuzzy network model is implemented in such a way that the adaptive neural network is used to tune the parameters of fuzzy inference system. Due to 
adaptive approach of ANFIS it can be used to classify the EEG signal very efficiently and the fuzzy layers are used to capture the stochastic nature of the EEG signals.

Besides that, the analysis of Takagi-Sugeno model based ANFIS shows that there is no constraint on the node functions of adaptive network except the piecewise differentiability and no constraint on the architecture except it would be feed-forward type. Due to this features, the compatibility and effectiveness of ANFIS model in case of non-stationary, complex and stochastic signal like EEG is quite apparent.

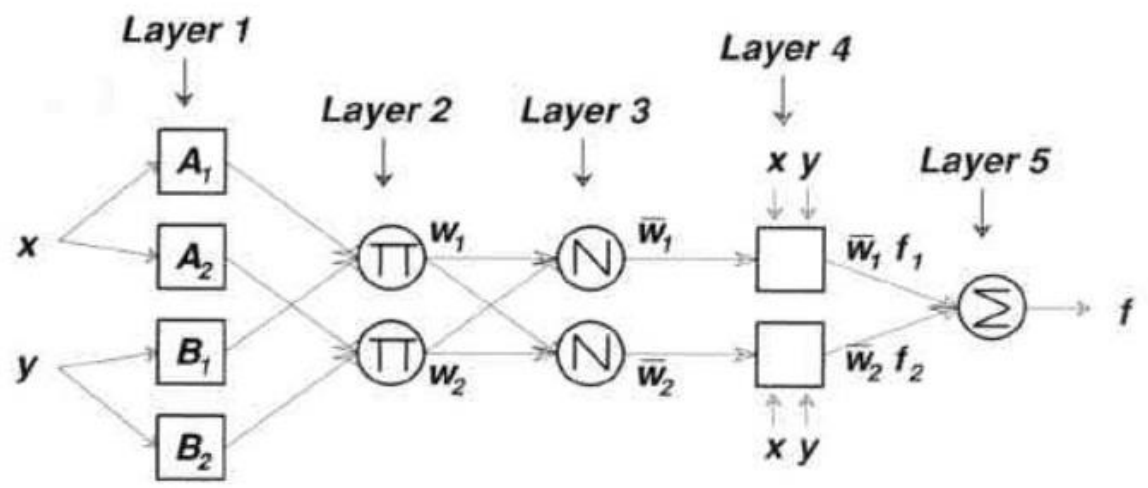

Fig. 8. Architecture of 5 layered ANFIS

Fig. 8 shows a prototype of the ANFIS used in our work. It contains five layers as described briefly below,

\subsubsection{Layer 1}

Every node in this layer is an adaptive node with a node function where $x$ (or $y$ ) is the input to node $I$ and $A_{i}$ (or $B_{i-2}$ ) is a linguistic label and output of the layer $O_{i}^{1}$ is the membership grade of fuzzy set $A$ (say $A_{1}, A_{2}, B_{l}$ or $B_{2}$ ) and it specifies the degree to which the given input $x$ (or $y$ ) satisfies the quantifier $A$.

$$
O_{i}^{1}=\mu_{A_{i}}(x)
$$

where, $x$ is the input of the $i^{\text {th }}$ node and $A_{i}$ is the linguistic variable associated with the transfer function of the corresponding node. Here, we have chosen the membership function as a normalized bell shaped curve, given by

$$
\mu_{A_{i}}(x)=\frac{1}{1+\left(\frac{x-c_{i}}{a_{i}}\right)^{2 b_{i}}}
$$

where, $a_{i}, b_{i}$ and $c_{i}$ are the parameters corresponding to the node function of the $i^{\text {th }}$ node. The parameters corresponding to this layer are called the premise parameters or antecedence parameters. 


\subsubsection{Layer 2}

Every node in this layer is a fixed node labelled $\Pi$, whose output is the product of all the incoming signals, where each node output represents the firing strength of a rule.

$$
O_{i}^{2}=w_{i}=\mu_{A_{i}}(x) \times \mu_{B_{i}}(y)
$$

where, $i=1,2, \ldots ., D$ and $\mathrm{D}$ is the dimension of the corresponding input vectors $\vec{x}$ and $\vec{y}$.

\subsubsection{Layer 3}

Every node in this layer is a fixed node labelled N, as shown in Fig. 8. The $i^{\text {th }}$ node of this layer normalizes the firing strength of the previous node with respect to firing strengths of others.

\subsubsection{Layer 4}

$$
O_{i}^{3}=\overline{w_{i}}=\frac{w_{i}}{\sum_{i} w_{i}}
$$

Every node in this layer is an adaptive node shown as square nodes in Fig. 8. The output of this node is given by

$$
O_{i}^{4}=\overline{w_{i}} f_{i}=\overline{w_{i}}\left(p_{i} x+q_{i} y+r_{i}\right)
$$

where, $\bar{w}_{i}$ is the output of $i^{\text {th }}$ node of layer 3 and $\left\{p_{i}, q_{i}, r_{i}\right\}$ are referred as the consequent parameters.

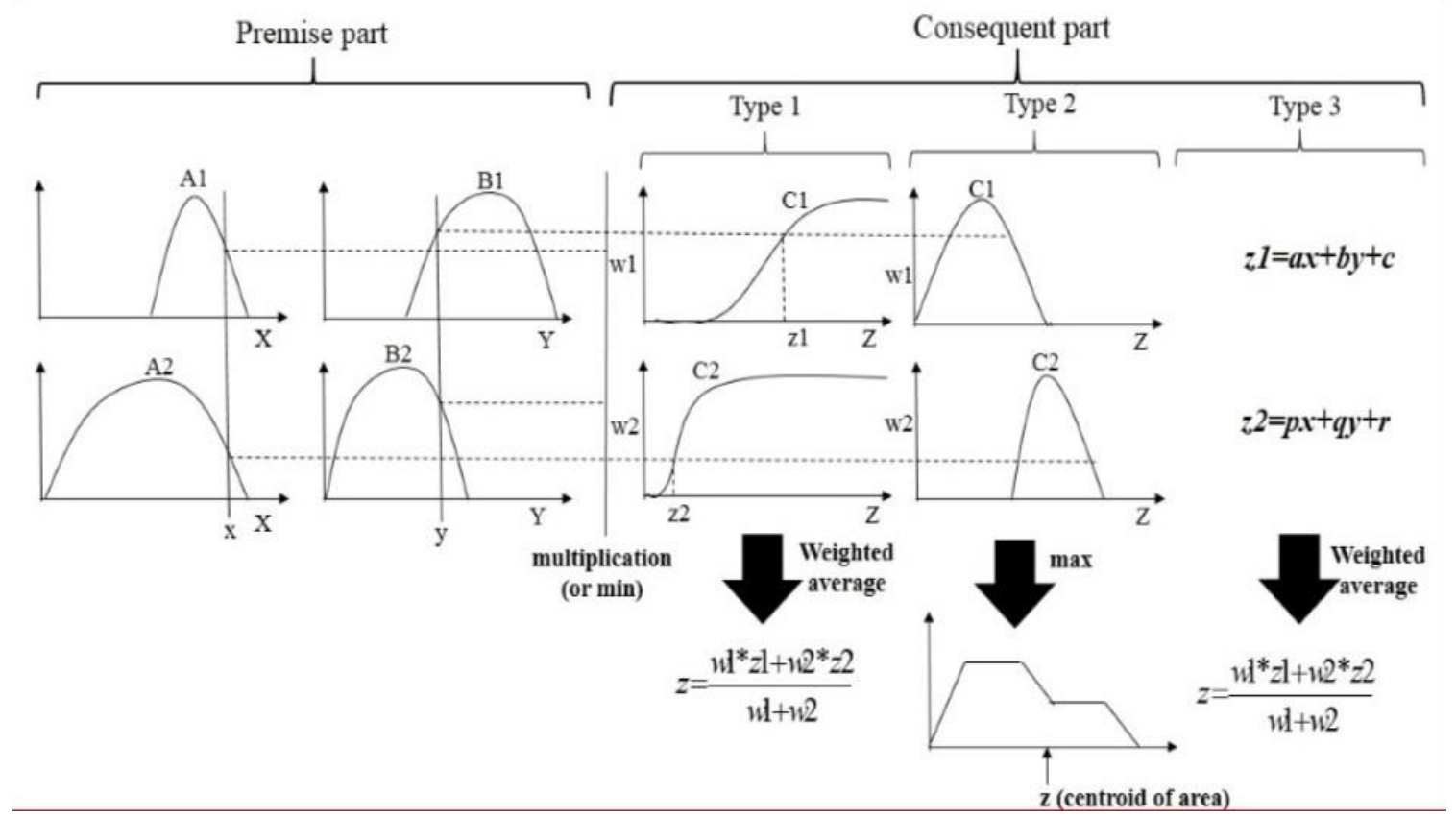

Fig. 9. Fuzzy reasoning mechanism for ANFIS

\subsubsection{Layer 5}

The single node in this layer is a fixed node which acts as an accumulator and it adds up all the outputs coming from the previous layer to give the final classifier function as, 


$$
O_{i}^{5}=\sum_{i} \bar{w}_{i} f_{i}=\frac{\sum_{i} w_{i} f_{i}}{\sum_{i} w_{i}}
$$

Thus, by using the above shown architecture we have constructed an adaptive network which is functionally equivalent to a type-3 fuzzy inference system. The operation of the ANFIS as a type-3 fuzzy inference system and the corresponding mappings are shown in Fig. 9 .

\subsection{Interval Type-2 Fuzzy Inference System}

In the path of evolution of sets and logic, it was seen that classical sets with their binary membership functions are unable to correspond the human knowledge with the inference systems used to control different processes or logic systems. From this perspective, Zadeh in [31] proposed fuzzy sets and logic where each of the fuzzy sets. These fuzzy sets imitate the human thought process to handle the uncertainties involved in the input-output system of fuzzy inference system. Each of the fuzzy sets contains a continuous membership function which describes the possibility of a number to be a member of that fuzzy set. This approach showed its effectiveness as an inference system for vast number of applications. But as researchers investigate more about the working procedure and uncertainty handling property of fuzzy sets, it became quite clear the uncertainty handling property of these type- 1 or classical fuzzy sets is not up to the mark as type- 1 fuzzy sets handle uncertainties by defining precise and crisp membership functions [32]. Therefore, the way to define MFs in type-1 fuzzy logic system (FLS) restricts the ability of type-1fuzzy sets and FLS to model and minimize the effect of uncertainties. This problem is actually faced when Type-1 ANFIS is implemented on non-stationary and uncertain systems like EEG.

A type-2 FLS has the potential to outperform a type-1 FLS because a type-2 fuzzy set is represented by more parameters than a type-1 fuzzy set [33]. Unlike a type-1 fuzzy set whose membership function (MF) is defined precisely, the MF of a type-2 fuzzy set is defined blurrily and consisted of a set of admissible type-1 MFs called the footprint of uncertainty (FOU) of a type-2 MF [34].Once a type-2 MF is reduced to a type-1 MF, the blurriness of the MF will no longer exist and it becomes a precise MF as defined in a type-1 FLS. Therefore, type-2 fuzzy logic can be viewed as a generalization of type-1 fuzzy logic, or on the other hand, type-2 fuzzy sets and MFs can also be considered as an extension of type-1 fuzzy sets and MFs with the increased ability to handle uncertainties existing in MFs and FLS. 


\subsection{Interval type-2 Fuzzy based Multiclass ANFIS algorithm}

In our proposed classification technique, binary ANFIS classifiers are used as the basic components of the OVA and OVO approach. Here, an interval type-2 fuzzy logic is used to combine the different outputs of the ANFIS classifiers to produce a final optimal result. The amalgamation of the three techniques, is coined as Interval Type-2 Fuzzy Logic ANFIS fusion (IT2FLF-ANFIS) algorithm. While fusing the results of each individual binary classifier, it is observed that different classes have their own distribution around hyper-planes and also the accuracy of classification between same two classes may vary depending on problems. This uncertainty involved in the variance of error and bias while constructing hyper-planes inspired us to use type-2 fuzzy logic fusion methods. Here, the type-2 fuzzy fusion block is adopted by us to unite all the piecewise hyper-planes to construct an efficient one with least discrepancies and regions of conflicts because type-2 fuzzy sets are more efficient in handling uncertainties than type-1 fuzzy sets [35]. The implementation of interval type-2 in the two variant approaches: OVA and OVO are explained in the following sub-sections.

\subsubsection{One versus All - Interval Type-2 fuzzy logic-ANFIS fusion (OVA-IT2FLF-ANFIS)} As mentioned earlier in section 4.1, the OVA approach needs $N$ binary ANFIS classifiers to discriminate each classes from corresponding $(N-1)$ classes. The $i^{\text {th }}$ classifier constructs the hyperplane $f_{i}$ and $N$ such $f_{i}$ 's are combined to generate the final output.

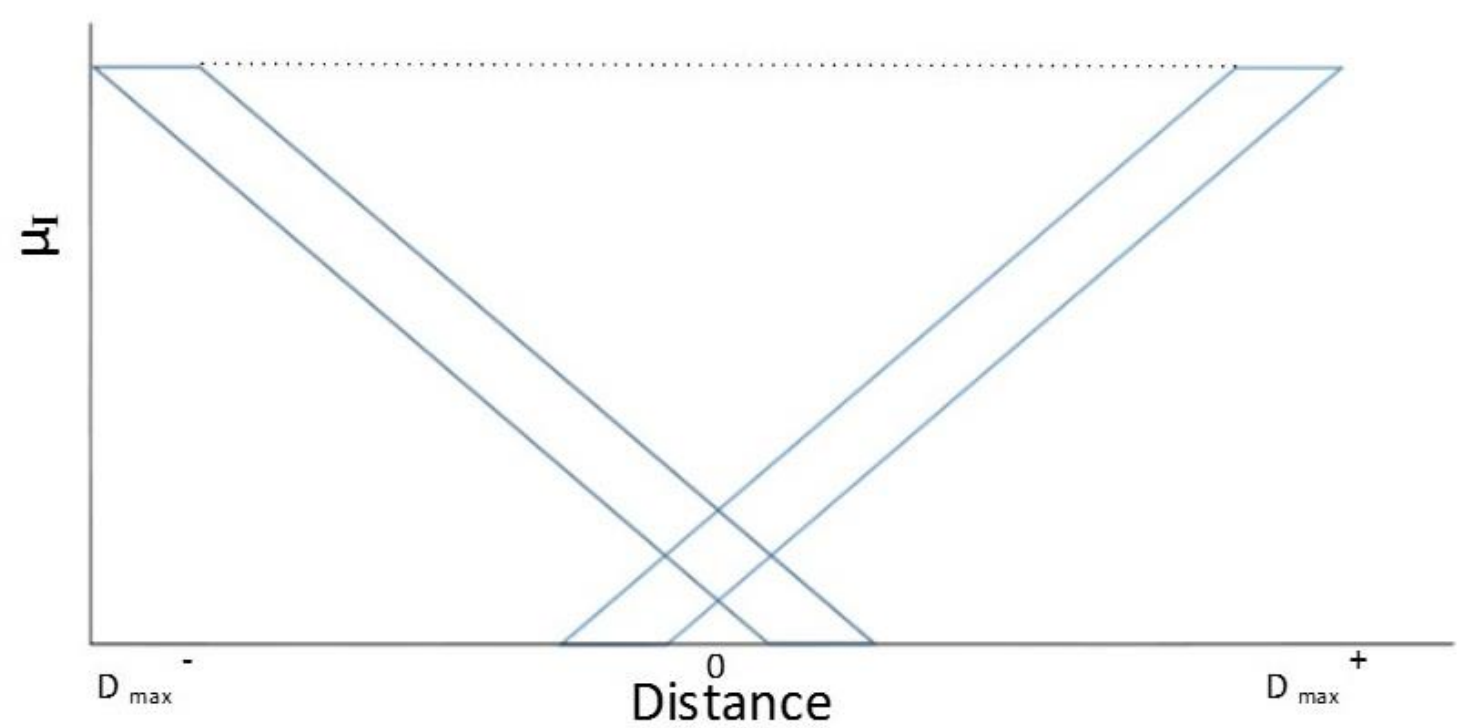

Fig. 10. Ideal representation of the membership functions distribution for distance as inputs to IT2FL inference engine

In $O V A-I T 2 F L F-A N F I S$ approach, the type-2 fuzzy sets fuses the outputs of each individual classifier and its distance from its corresponding hyper-plane $f_{i}$. For example, say, a data point $x$ belongs to class 1 , then its distance from its corresponding hyperplane, say, $f_{l}$ should be positive or zero. On the contrary, if the distance is negative, than $x$ will not belong to class 
1. Now, as the distance becomes more positive, the possibility that $x$ will belong to class $i$ will increase and would be independent of the discrepancies introduced by other $(N-1)$ classifiers. Thus, the discrete classifier output and the distance from each individual ANFIS classifier are used to construct the type-2 fuzzy sets. Here, we have set the base point of positive and negative membership function (MF) around -0.25 and 0.25 , respectively (as illustrated in Fig. 10). The admissible range of the corresponding type-2 MFs is set to 0.1-0.2.

As the system has $\mathrm{N}$ distinct inputs from each of the $\mathrm{N}$ classifiers and each of the inputs have two possibilities, there will be $2 N$ fuzzy rules where the $i^{\text {th }}$ rule is defined as,

$$
\begin{aligned}
& \text { IF } x \text { is } A_{1}, x \text { is } A_{2}, \ldots, x \text { is } A_{N}, \\
& \text { THEN } x \text { is } O_{i}
\end{aligned}
$$

where, $A_{1}, A_{2}, \ldots, A_{N}$ is $\{$ negative or positive $\}$ classifier output or distance values and $\mathrm{O}_{\mathrm{i}}$ in $\left\{\mathrm{O}_{1}, \ldots, \mathrm{O}_{\mathrm{N}}\right\}$, which is the set of all possible output sets. For simplicity, let the consequences of these fuzzy rules consider the distance inputs of $N$ classifiers. Thus, if for one data input $\mathrm{A}_{1}$ yields a 'positive' value and the other classifiers produces 'negative' value, then the consequence of the corresponding fuzzy rule will be the output fuzzy set $\mathrm{O}_{1}$. Based on the same considerations the consequences of other rules will occur. In the inference part of the fuzzy model, we use the product t-norm operation and join it under the max operation and supstar composition [36, 37]. Now the resultant output type-2 fuzzy sets are de-fuzzified using the center of the set method. The centers of sets are calculated using Karnik-Mendel algorithm [38] and the iterative algorithm [37]. The corresponding crisp values will be in the range $[1, N]$, which are rounded up to the nearest integer and the final output signify the class in which the data point belongs.

\subsubsection{One versus One - Interval Type-2 fuzzy logic-ANFIS fusion (OVO-IT2FLF-ANFIS)}

To implement the OVO approach, the outputs of $N(N-1) / 2$ binary ANFIS classifiers are required to get the final output (see Section 4.1). In case of designing the interval type-2 fuzzy logic based fusion algorithm for OVO (OVO-IT2FLF-ANFIS), distance along with the binary classifier output does not qualify as sufficient inputs to the inference engine. As the decisions generated by each of the classifiers sometimes may lead to confusions due to unwanted overlap between the zones segregated by the classifiers. Thus, the distances of the data point from the centroids of each of the classes are also considered as another input. These distances help the fusion system to identify the data points which belong to the region of confusion, which actually belongs to one of the $\mathrm{N}$ classes. The classification system used in OVO, which is a bit different from OVA can be mapped with fuzzy linguistic variables 
[37] distance from the hyper-planes and centroidal distances. The base points of the MFs for the distance values have been set similar to the one used in OVA-IT2FLF-ANFIS classifier, as the principles to determine the class of an unknown data point using the distance information is the same. Similar to the construction of the distance MFs, here, the centroidal distance can take two forms- near and far, whose ideal representation is shown in Fig. 11. When the distance of a certain data point form the centroid of a certain class becomes 'near', the possibility that it will belong to that class will be highest and as it becomes 'far', it can be concluded that the data point does not belong to the corresponding class.

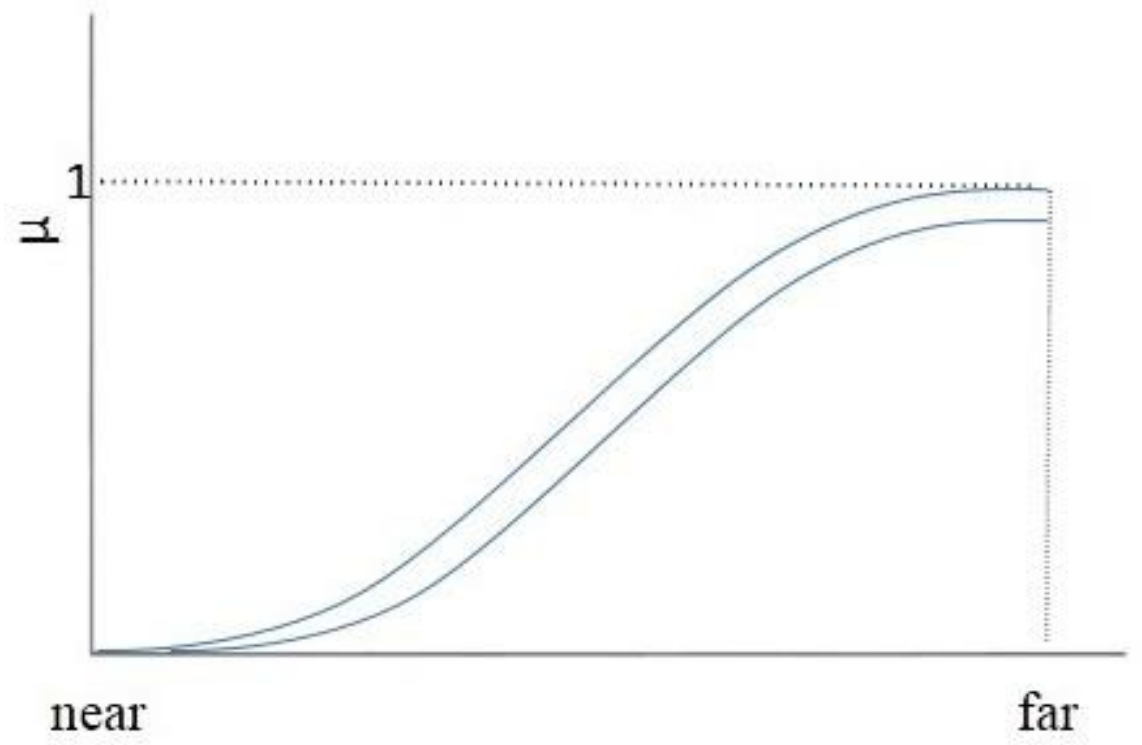

\section{Centroidal Distance}

Fig. 11. Ideal representation of the membership functions distribution for centroidal distance as inputs to IT2FL inference engine

As the system has $\mathrm{N}$ distinct inputs from each of the $\mathrm{N}$ classifiers and each of the inputs have two possibilities, there will be $2^{\mathrm{N}(\mathrm{N}-1) / 2}$ fuzzy rules where the $i^{\text {th }}$ rule can be defined as,

$$
\begin{aligned}
& \text { IF } x \text { is } \mathrm{A}_{1}, x \text { is } \mathrm{A}_{2}, \ldots, x \text { is } \mathrm{A}_{\mathrm{N}}, x \text { is } \mathrm{C}_{1}, x \text { is } \mathrm{C}_{2}, \ldots, x \text { is } \mathrm{C}_{\mathrm{N}} \\
& \text { THEN } x \text { is } \mathrm{O}_{\mathrm{i}}
\end{aligned}
$$

where, $A_{1}, A_{2}, \ldots, A_{N}$ is $\{$ negative or positive $\}$ classifier output or distance values, $C_{1}, C_{2}$, $\ldots, \mathrm{C}_{\mathrm{N}}$ is $\{$ near or far $\}$ values and $\mathrm{O}_{\mathrm{i}}$ in $\left\{\mathrm{O}_{1}, \ldots, \mathrm{O}_{\mathrm{N}}\right\}$, which is the set of all possible output sets.

The consequences of these fuzzy rules consider the binary classifier output and distance inputs of $\mathrm{N}$ classifiers and centroidal distances of data point from centroids of $\mathrm{N}$ classes. For example, if for one data input, if $\mathrm{A}_{1}$ classifies an output as 'positive' and $\mathrm{C}_{1}$ classifies an output as 'near', and the rest of the A and C classifiers yield 'negative' and 'far' output, 
respectively, then the consequence of the corresponding fuzzy rule will be the output fuzzy set $\mathrm{O}_{1}$. The other fuzzy rules will also yield the corresponding consequences on a similar basis.

Now, the final inference engine and the defuzzifier or output processing block [39] of the used fuzzy fusion model OVA-IT2FLF-ANFIS, is a replicate of the inference and defuzzifier unit of $O V O-I T 2 F L F-A N F I S$. So, here too, we get an integer in the range $[1, \mathrm{~N}]$ signifying the class in which the data point belongs.

\section{RESULTS AND DISCUSSION}

The results of our proposed multi-class classification algorithm and our robot control strategy for 11 subjects is discussed in this section. First, we describe the performance of the two variants of our multiclass IT2FLF-ANFIS classifier: OVA-IT2FLF-ANFIS and OVOIT2FLF-ANFIS and provides a statistical comparison with the following standard classifiers: Linear Discriminant Analysis (LDA), k-Nearest Neighbor (kNN), Support Vector Machine (SVM) and Naïve Bayesian (NB) [40, 41] using both OVA and OVO method for multi-class classification. Then, in the following section we examine the performance of our online control strategy using both the proposed classifiers.

The processing and detection of the mental states from the EEG signals has been done in MATLAB 2012b platform run on a computer with the following specifications: Intel core i7 processor@ 3.25 clock speed, 8GB RAM and 64 bit Windows 7 operating system.

\subsection{Offline training}

The feature vector used for training the classifiers are prepared from the probability distribution of the local Hurst component (as mentioned in Section 3.3) from each session (of each subject). As each 7 session is made of 100 trials of data, then the final size of the feature vector is 700 (trials) $\times 14$ (electrodes) $\times 45$ (features). The total feature vector is then divided into two separate datasets: training and validation, using k-fold cross validation technique [41]. The performance of the classifiers are determined by the values obtained by the average of two metrics: classification accuracy (C.A.) and area under the ROC curve (AUC) [42], over 10 runs $(k=10)$.

The C.A. and AUC of the two proposed OVA-T2FLF-ANFIS and OVO-IT2FLF-ANFIS classifiers are given in Table. 2. As noted from the table, the recognition rate for the five mental states: Forward, Backward, Left, Right and No movement is more than $80 \%$ for both the variants of the classifier with Subject 1 producing the best result for both the classifiers 
(OVA-T2FLF-ANFIS: C.A. $=96 \%, \quad$ AUC $=92.86 \%$ and OVO-IT2FLF-ANFIS: C.A. $=$ $99.50 \%, \mathrm{AUC}=97.37 \%$ ). It is also noted that OVO method performs slightly better than OVA approach in terms of classification accuracy.

Table 2. Offline performance analysis of the proposed OVA-IT2FLF-ANFIS and OVO-IT2FLF-ANFIS classifier for 11 subjects

\begin{tabular}{lllll}
\hline \multirow{2}{*}{ Subject ID } & \multicolumn{2}{l}{ OVA-IT2FLF-ANFIS } & \multicolumn{2}{l}{ OVO-IT2FLF-ANFIS } \\
\cline { 2 - 5 } & C.A. & AUC & C.A. & AUC \\
\hline 1 & 96.00 & 92.86 & 99.50 & 97.37 \\
2 & 86.00 & 82.50 & 88.00 & 80.70 \\
3 & 80.00 & 80.00 & 80.00 & 80.00 \\
4 & 86.00 & 85.45 & 87.50 & 87.67 \\
5 & 92.50 & 90.00 & 90.00 & 90.00 \\
6 & 89.25 & 89.25 & 94.50 & 92.34 \\
7 & 90.00 & 89.44 & 90.00 & 88.67 \\
8 & 85.00 & 83.23 & 92.00 & 88.00 \\
9 & 87.50 & 85.00 & 88.50 & 85.00 \\
10 & 94.50 & 92.10 & 96.75 & 92.53 \\
11 & 91.25 & 90.00 & 93.50 & 93.50 \\
\hline Mean & 88.91 & 87.26 & 90.93 & 88.71 \\
\hline
\end{tabular}

The performance of our proposed OVA-T2FLF-ANFIS and OVO-IT2FLF-ANFIS classifier combination has been compared with its competitors: LDA, kNN, SVM and NB using Friedman Test [43]. To maintain parity in the comparison process, multi-class classification of the competitor algorithms are also done using OVA and OVO approach.

According to the null hypothesis in this context, all the classifiers are equivalent and hence their ranks $R_{j}$ should be equal. The Friedman statistic is given by

$$
\chi_{F}^{2}=\frac{12 N}{k(k+1)}\left[\sum_{j} R_{j}^{2}-\frac{k(k+1)^{2}}{4}\right]
$$

with $k-1$ degrees of freedom is distributed accordingly to $\chi_{F}^{2}$ with $k-1$ degrees of freedom, where $k$ is the number of algorithms to be compared and $N$ is the number of parameters used for comparison. In this study, the mean of the classification accuracy (over 11 subjects) is 
considered as the number of parameters, thus, $N=1$ and $k$ is the number of classification algorithms which is 10 .

Table 3 provides the mean classification accuracy for the classification techniques and their corresponding ranks based on their accuracy. Using the ranks $R_{j}$ from Table $3, \chi_{F}^{2}$ is calculated as 8.945 for both the features which is greater than $\chi_{9,0.95}^{2}=3.325$. This means that for $(\mathrm{k}-1=10-1=) 9$ degrees of freedom one can say the null hypothesis is wrong for a confidence level of $95 \%$ and hence, the classifiers are not equivalent rather they are ranked according to $R_{j}$. This justifies our claim of using our proposed algorithms as the classifier rather than other standard classifiers, in this study.

Table 3. Statistical validation of the proposed OVA-IT2FLF-ANFIS and OVOIT2FLF-ANFIS using Friedman test

\begin{tabular}{lll}
\hline Classifier Algorithm & Classification Accuracy & Rank $\left(\mathbf{R}_{\mathbf{j}}\right)$ \\
\hline OVA-IT2FLF-ANFIS & 88.91 & 2 \\
OVO-IT2FLF-ANFIS & 90.93 & 1 \\
OVA-LDA & 78.57 & 10 \\
OVO-LDA & 79.43 & 9 \\
OVA-KNN & 82.67 & 8 \\
OVO-KNN & 82.13 & 7 \\
OVA-SVM & 85.16 & 6 \\
OVO-SVM & 86.25 & 3 \\
OVA-NB & 85.75 & 4.5 \\
OVO-NB & 85.75 & 4.5 \\
\hline
\end{tabular}

\subsection{Real-time robot arm control performance (Online testing)}

Following the training of the classifiers, the system is ready to perform online control of the Jaco robot arm. The setup of the online experimentation is shown in Fig. 12(a) where the yellow ball is target position. The subject controls the directional motion of the robot arm using the mental commands, mentioned in Table 1. The subject performed this experiment over 20 runs using both the proposed algorithm. In each run, the subject would attempt to reach the target using the robot arm. During real-time testing, there was no time constraint imposed on the subject and he/she would attempt to reach the target in his/her own time. Each run ended with the robot arm either reaching the target or the subject giving up in 
between.

The online performance of our proposed classifiers are determined by the following metric: i) percentage (\%) success rate, and ii) Information Transfer Rate (ITR) [44]. The \% success rate is defined by the number of times the subject was successful in reaching the target within a positional error of $5 \%$ with relation to the target. ITR $\left(B_{t}\right)$ represents the bit rate of the method. Its representation in bits/min is given as

$$
B_{t}=\left(\log _{2} N+P \log _{2} P+(1-P) \log _{2} \frac{1-P}{N-1}\right) \times \frac{60}{T}
$$

where, $N$ represents the number of possible states which is 5 in the present context and $P$ represents the classification accuracy between 0 and $1 . T$ is the time needed to convey each action in second/symbol i.e., time interval from the issue of a command to the classified output of the same.

The $\%$ success rate and ITR for the two proposed classifier for 11 subjects are shown in Table 4. Maintaining parity with the performance during offline training, here too, the OVOIT2FLF-ANFIS performs better than the OVA-IT2FLF-ANFIS in terms of their success rate. The best result is given by Subject 1 where he reaches the target (within 5\% error) $80 \%$ of the time, i.e., 18 times over 20 runs with an ITR of 33.70 bits/min.

It is noted from Table 4 that OVO approach takes much longer to produce an output than OVA. Such wide difference in computation may be attributed to the large number of subclassifiers the OVO approach employs to yield a result compared to that of OVA (mentioned in Section 4.1). Table 4 also includes the average time taken by the subject to reach the target. Snapshots of a subject performing the experiment to reach the target using motor imagery signals are shown in Fig. 12.

\section{CONCLUSION AND FUTURE DIRECTION}

In this paper, two variants of multi-class classification algorithm: OVA-IT2FLF-ANFIS and OVO-IT2FLF-ANFIS have been developed towards recognition of motor imagery mental states in real time. For this purpose, we devised an experiment in which the subject would generate five mental commands: forward, backward, left, right and no movement and employed multi-fractal detrended fluctuation analysis to create the feature vector. In the initial stages of the experiment, the subjects and proposed classifiers are trained and the performance of the training is determined by the classification accuracy and area under the ROC curve. An average training accuracy of $88.91 \%$ and $90.93 \%$ are obtained from 11 
subjects for the OVA-IT2FLF-ANFIS and OVO-IT2FLF-ANFIS algorithm, respectively. The proposed classifiers have also been statistically validated using Friedman Test.

Table 4. Online performance analysis of the proposed OVA-IT2FLF-ANFIS and

OVO-IT2FLF-ANFIS classifier for 11 subjects

\begin{tabular}{|c|c|c|c|c|}
\hline \multirow{2}{*}{ Subject ID } & \multicolumn{2}{|c|}{ OVA-IT2FLF-ANFIS } & \multicolumn{2}{|c|}{ OVO-IT2FLF-ANFIS } \\
\hline & \% success rate & ITR & $\%$ success rate & ITR \\
\hline 1 & 75.00 & 23.47 & 80.00 & 33.70 \\
\hline 2 & 60.00 & 22.97 & 80.00 & 33.11 \\
\hline 3 & 50.00 & 24.42 & 60.00 & 33.74 \\
\hline 4 & 60.00 & 24.38 & 65.00 & 32.22 \\
\hline 5 & 60.00 & 23.93 & 70.00 & 32.30 \\
\hline 6 & 65.00 & 23.83 & 65.00 & 33.01 \\
\hline 7 & 70.00 & 22.53 & 75.00 & 33.74 \\
\hline 8 & 70.00 & 24.13 & 70.00 & 32.50 \\
\hline 9 & 60.00 & 22.30 & 65.00 & 32.25 \\
\hline 10 & 70.00 & 24.00 & 65.00 & 33.15 \\
\hline 11 & 70.00 & 22.10 & 75.00 & 33.12 \\
\hline Mean & 64.50 & 23.46 & 70.00 & 32.98 \\
\hline $\begin{array}{l}\text { Average Time } \\
\text { Taken by the } \\
\text { classifier }\end{array}$ & \multicolumn{2}{|c|}{137 seconds } & \multicolumn{2}{|c|}{198 seconds } \\
\hline $\begin{array}{l}\text { Average Time } \\
\text { Taken to reach } \\
\text { target by the } \\
\text { subjects }\end{array}$ & \multicolumn{2}{|c|}{685 seconds } & \multicolumn{2}{|c|}{916 seconds } \\
\hline
\end{tabular}

The performance of the real time control is defined by the percent success rate of the robot arm reaching the target and information transfer rate. The average success rate obtained for the 11 subjects are $64.5 \%$ (i.e., approximately 13 successful hits for a total 20 runs) and $70 \%$ (i.e., 14 successful hits for a total 20 runs) for the OVA-IT2FLF-ANFIS and OVOIT2FLF-ANFIS algorithm, respectively. The average ITR over 20 runs for 11 subjects is $23.46 \mathrm{bits} / \mathrm{min}$ and $32.98 \mathrm{bits} / \mathrm{min}$ for the OVA-IT2FLF-ANFIS and OVO-IT2FLF-ANFIS algorithm, respectively. These results suggests that our proposed IT2FLF-ANFIS approach 
towards multi-class detection is efficient in dealing with non-stationary and uncertain signal data classification like EEG. Even though OVO-IT2FLF-ANFIS performs better than OVAIT2FLF-ANFIS, implementation of this approach for real time scenario is not feasible because of the high computational time the method takes to yield an output. Thus, the OVA approach is the preferred method for real time cases.

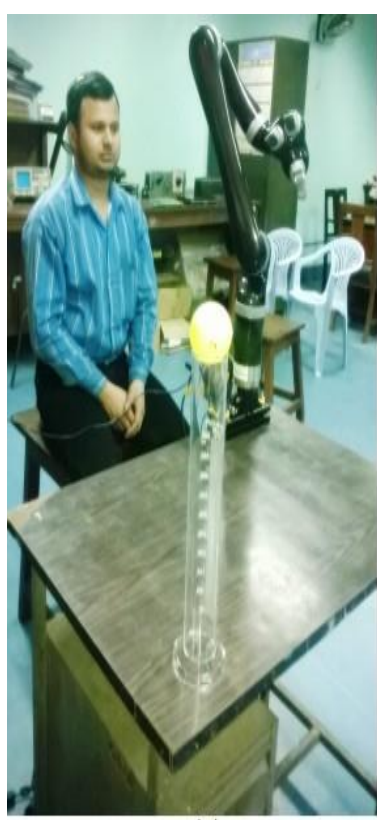

(a)

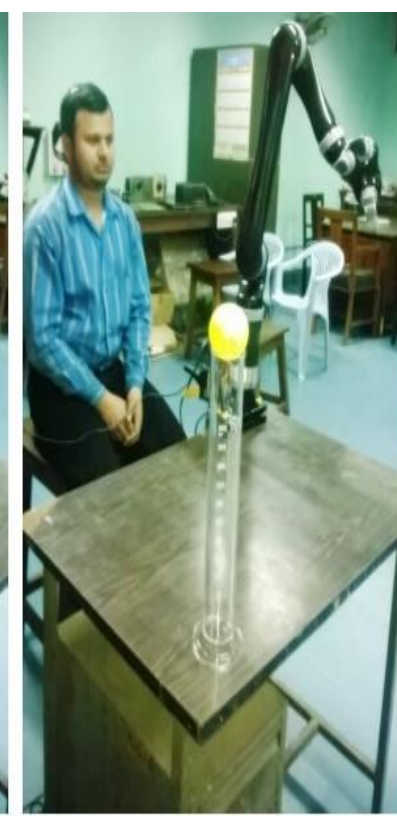

(b)

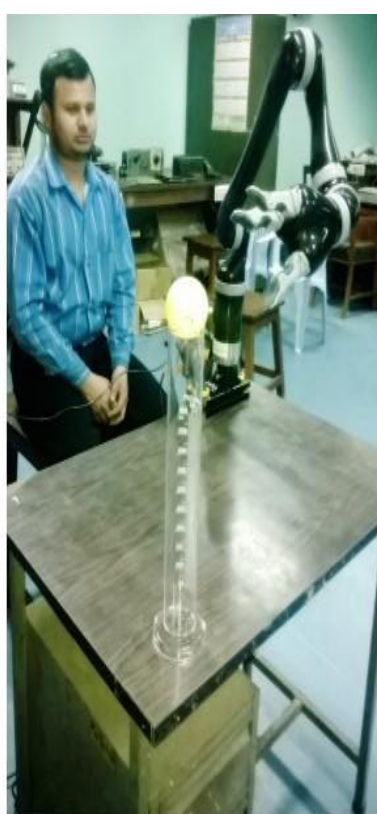

(c)

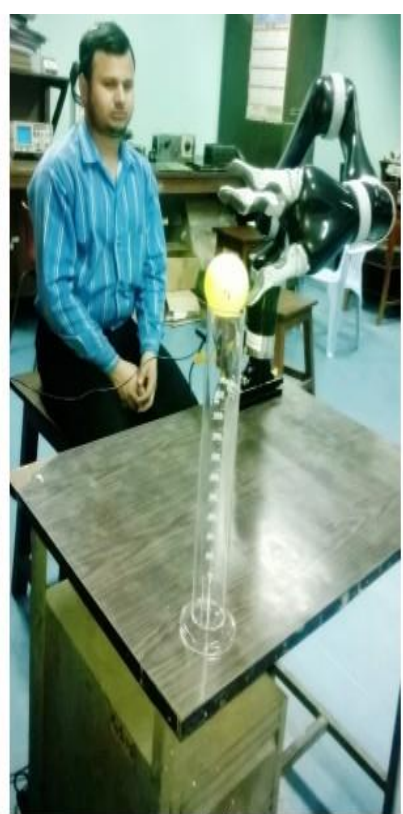

(d)

Fig. 12. Illustrations of a subject attempting to move towards the target (yellow ball). (a) initial position of the robot arm, (b) the subject moves the arm forward to align it with the target, (c) the subject moves the robot in clockwise direction (mental command: right), (d) the subject again moves the robot in clockwise direction (mental command: right) and finally reaches the target

The pros and cons of the methods make it evident that in spite of using the OVA and OVO methods differently as two separate classifiers, we may cluster their results using some fuzzy rule base which will make a trade-off between all the multi class handling methods and will give a better result with comparably lower computational complexity. Here, we have used only least mean square algorithm for parameter handling of OVO-IT2FLF-ANFIS and OVA-IT2FLF-ANFIS but we may replace it by some better optimization algorithms and meta-heuristics. So we have a rich future perspective to work with the proposed algorithm. Also, in our future studies, we would attempt to control the individual links of the robot arm using motor imagery EEG and move towards the development of a BCI-controlled prosthetic device for commercial use.

\section{Acknowledgements.}


I would like to thank University Grants Commission, India, University of Potential

Excellence Programme (Phase II) in Cognitive Science, Jadavpur University and Council of Scientific and Industrial Research, India.

\section{References}

1. S. Keates, P. Varker, F. Spowart, Human-machine design considerations in advanced machinelearning systems, IBM J. Rsch. \& Dev. 55 (5) (2011) 4:1-4:10.

2. D. J. McFarland, J. R. Wolpaw, Brain-computer interface operation of robotic and prosthetic devices, Computer 41(10) (2008) 52-56.

3. C. Breitwieser, I. Daly, C. Neuper, G.R. Muller-Putz, Proposing a standardized protocol for raw biosignal transmission, IEEE Trans. Biomed. Eng. 59(3) (2012) 852-859.

4. S. Bermudez i Badia, A.G. Morgade, H. Samaha, P.F.M.J. Verschure, Using a Hybrid Brain Computer Interface and Virtual Reality System to Monitor and Promote Cortical Reorganization through Motor Activity and Motor Imagery Training. IEEE Trans. Neural Sys. Rehab. Eng. 21 (2) (2013) 174-181.

5. S. Bordoloi, U. Sharmah, S.M. Hazarika, Motor imagery based BCI for a maze game. In: $4^{\text {th }}$ Int. Conf. Intelligent Human Computer Interaction (IHCI),Kharagpur, India (2012) 1-6.

6. S. Bhattacharyya, A. Sengupta, T. Chakraborti, D. Banerjee, A. Khasnobish, A. Konar, D.N. Tibarewala, R. Janarthanan, EEG controlled remote robotic system from motor imagery classification. In: $3^{\text {rd }}$ Int. conf on Com., Comm. \& Networking Tech. (ICCCNT 2012), Chennai, India (2012) 1-8.

7. Y. Chae, J. Jeong, S. Jo, Toward brain-actuated humanoid robots: Asynchronous direct control using an EEG-based BCI, IEEE Trans. Robotics 28(5) (2012) 1131-1144.

8. J.J. Daly, J.R. Wolpaw, Brain-computer interfaces in neurological rehabilitation, Lancet Neurol 7 (2008) 1032-43.

9. A.B. Schwartz, X.T. Cui, D.J. Weber, D.W. Moran, Brain Controlled Interfaces: Movement Restoration using Neural Prosthetics, Neuron 52 (2006) 205-220.

10. G. Dornhege, Towards Brain-Computer Interfacing, first ed., MIT Press, Cambridge, MA, 2007.

11. G. Schalk, Sensor modalities for brain-computer interfacing, Human-Computer Interac. Novel Interact. Methods Tech. Lec. Notes Comp. Sc. 5611 (2009) 616-622.

12. S. Mason, A. Bashashati, M. Fatourechi, K. Navarro, G. Birch, A comprehensive survey of brain interface technology designs, Ann. Biomed. Eng. 35 (2007) 137-169.

13. J.R. Millán, R. Rupp, G.R. Müller-Putz, R. Murray-Smith, C. Giugliemma, M. Tangermann, C. Vidaurre, F. Cincotti, A. Kübler, R. Leeb, C. Neuper, K.R. Müller, and D. Mattia, “Combining brain-computer interfaces and assistive technogies: State-of-the-art and challenges," Front. Neurosci. 4 (2010) 1-15. 
14. S. Nieuwenhuis, K.R. Ridderinkhof, J. Blom, G.P.H. Band, A. Kok, Error-related potentials are differentially related to awareness of response errors: Evidence from an antisaccade task, Psychophysiology 38 (2001) 752-760.

15. G. Pfurtscheller, F.H. Lopes da Silva, Event-related EEG/MEG synchronization and desynchronization: Basic principles, Clin. Neurophysio. 110 (11) (1999) 1842-1857.

16. C. Qiang, P. Hu, F. Huanqing, Experiment study of the relation between motion complexity and event-related desynchronization/synchronization, In: 1st Int. Conf. Neural Interface \& Cont. (2005), 14-16.

17. J. Friedman, Another approach to polychotomous classification, Technical report, Department of Statistics, Stanford University, 1996.

18. C. Bishop, Neural Networks for Pattern Recognition, first ed., Oxford University Press, Oxford, 1996.

19. J-S.R., Jang, ANFIS: Adaptive-network-based fuzzy inference system, IEEE Trans Syst. Man Cybern. 23(3) (1993) 665-685.

20. I. Svalina, V. Galzina, R. Lujic, G. Simunovic, An adaptive network-based fuzzy inference system (ANFIS) for the forecasting: The case of close price indices, Expert Sys. With App. 40 (15) (2013), 6055-6063.

21. N.N. Karnik, J.M. Mendel (1998) An introduction to type-2 fuzzy logic systems, In: IEEE The 1998 IEEE Int. Conf. Fuzzy Sys. Proc., Anchorage, AK, (1998) 915-920.

22. G. Prasad, P. Herman, T.M. McGinnity, Critical observations on type-2 fuzzy logic approach to uncertainty handling in a Brain-Computer Interface design. In: $11^{\text {th }}$ Int. Conf. Information Process. \& Management of Uncertainty, Paris (2006) 8pp.

23. L. Bougrain, O. Rochel, O. Boussaton, L. Havet, From the decoding of cortical activities to the control of a Jaco robot arm: A whole processing chain, Contr. Arch. Robotics CoRR abs/1212.0083 (2012).

24. V. Maheu, J. Frappier, P.S. Archambault, F. Routhier, Evaluation of the JACO robotic arm: Clinico-economic study for powered wheelchair users with upper-extremity disabilities," In: IEEE Int. Conf. Rehab. Rob. (ICORR) (2011) 1-5.

25. S. Sanei, J.A. Chambers, EEG Signal Processing, first ed., Wiley, West Sussex, 2007.

26. R. Palaniappan, Brain computer interface design using band powers extracted during mental tasks. In: 2nd International IEEE EMBS Conference on Neural Engineering (2005) 321-324.

27. J.W. Kantelhardt, S.A. Zschiegner, E. Koscielny-Bunde, A. Bunde, S. Havlin, H.E. Stanley, Multifractal detrended fluctuation analysis of nonstationary time series, Physica A- Statistical Mechanics \& its App. (2002).

28. E.A.F. Ilhen, Introduction to multifractal detrended fluctuation analysis in matlab, Front. Physiol. 3(141) (2012) doi: 10.3389/fphys.2012.00141. 
29. D.M.J. Tax, R.P.W. Duin, Using two-class classifiers for multiclass classification, In: $16^{\text {th }}$ Int. Conf. Pattern Recognition (2002) 124-127.

30. J. Roger, Adaptive-network-based fuzzy inference system, IEEE Trans. SMC 23 (3) (1993), 665-685.

31. L. Zadeh, Fuzzy sets, Information and Control 8 (1965) 338-353.

32. A. Abraham, B. de Baets, M. Koppen, N. Bertram (2006) Applied Soft Computing Technologies: The Challenge of Complexity. Springer 34 (2006) 320-321.

33. J. Zeng, Z.Q. Liu, Type-2 Fuzzy Sets for Pattern Recognition: The State-of-the-Art, Journal of Uncertain Systems 1 (3) (2007) 163-177.

34. J.M. Mendel, R.I. John, F. Liu, Interval type-2 fuzzy logic systems made simple, IEEE Trans. Fuzzy Sys. 14(6) (2006) 808-821.

35. D. Wu, J. M. Mendel, Uncertainty measures for interval type-2 fuzzy sets, Information Sciences 177 (2007) 5378-5393.

36. N. N. Karnik, J. M. Mendel, Operations on Type-2 Fuzzy Sets, Fuzzy Sets and Systems 122 (2001) 327-348.

37. H. Wu, J. M. Mendel, Uncertainty Bounds and Their Use in the Design of Interval Type-2 Fuzzy Logic Systems, IEEE Trans. on Fuzzy Systems 10 (2002) 622-639.

38. N. N. Karnik, J. M. Mendel, Centroid of a type-2 fuzzy set, Information Sciences 132 (2001) 195-220.

39. Q. Liang, J.M. Mendel, MPEG VBR video traffic modeling and classification using fuzzy technique, IEEE Trans. Fuzzy Systems 9 (1) (2001) 183-193.

40. R. O. Duda, P. E. Hart, D. G. Stork, Pattern Recognition, second ed., Wiley-Interscience, New York, 2001.

41. E. Alpaydin, Introduction to machine learning, second edition, MIT Press, Cambridge, 2009.

42. A.H. Fielding, J.F. Bell, A review of methods for the assessment of prediction errors in conservation presence/absence models, Environmental conservation 24(1) (1997) 38-49.

43. J. Demšar, Statistical comparisons of classifiers over multiple data sets, The J. of Mach. Lear. Res. 7 (2006) 1-30.

44. B. Obermaier, C. Neuper, C. Guger, G. Pfurtscheller, Information transfer rate in a five-classes brain-computer interface. IEEE Trans. Neural Sys. \& Rehab. Eng. 9(3) (2001) 283-288. 